\title{
SUPG-BASED STABILIZATION USING A SEPARATED REPRESENTATIONS APPROACH
}

\author{
D. González ${ }^{1 *}$ L. Debeugny ${ }^{1}$, E. Cueto ${ }^{1}$, F. Chinesta ${ }^{2}$, P. Díez ${ }^{3}$, A. Huerta ${ }^{3}$
}

\begin{abstract}
We have developed a new method for the construction of Streamline Upwind Petrov Galerkin (SUPG) stabilization techniques for the resolution of convection-diffusion equations based on the use of separated representations inside the Proper Generalized Decompositions (PGD) framework. The use of SUPG schemes produces a consistent stabilization adding a parameter to all the terms of the equation (not only the convective one). SUPG obtains an exact solution for problems in 1D, nevertheless, a generalization does not exist for elements of high order or for any system of convection-diffusion equations. We introduce in this paper a method that achieves stabilization in the context of Proper Generalzied Decomposition (PGD). This class of approximations use a representation of the solution by means of the sum of a finite number of terms of separable functions. Thus it is possible to use the technique of separation of variables in the context of problems of convection-diffusion that will lead to a sequence of problems in 1D where the parameter of stabilization is well known.
\end{abstract}

KEYWORDS: Convection-diffusion equation, SUPG, Proper Generalized Decomposition, Separated Representations, Finite Sum Decomposition

\section{INTRODUCTION}

The main aim of this paper is present one application of a new numerical strategy able to circumvent some of the numerical difficulties due to multidimensional character of some convection-diffusion equations.

It is well-known that the behavior of standard Finite Element in the resolution of convection-diffusion(-reaction) is not good. The use of numerical stabilization terms in the differential equation has been developed y discussed since some years ago. The use of Streamline Upwind Petrov Galerkin (SUPG) stabilization technique offers exact solution in 1D problems, but don't work well in the application of this stabilization technique in problems on multidimensional spaces.

The use of the Proper Generalized Decompositions strategies and its character of one-dimensional products approximation provides us the possibility of develop this technique applying SUPG stabilization in the resolution of the convection-diffusion problems defined in multidimensional spaces.

The outline of the paper is as follows. In the next section we describe the framework of the class of convectiondiffusion(-reaction) problems we deal with. The PGD method used to apply the stabilization technique is described in the section 3 . In section 4 we describe some examples in order to show the behavior of the technique

\footnotetext{
*Corresponding author: gonzal@unizar.es
}

in the simulation of this class of problems.

\section{PROBLEM SETTING}

Only for simplicity we consider the steady-state convection-diffusion problem. The equation that govern this problem is given by

$$
\mathbf{a} \cdot \nabla u-\nabla \cdot(\nu \nabla u)=s \text { in } \Omega \subset \mathbf{R}^{n},
$$

with $n=2,3$ and with boundary conditions

$$
\begin{aligned}
u & =u_{D} \text { on } \Gamma_{D} \\
\mathbf{n} \cdot \nu \nabla u & =\nu \frac{\partial u}{\partial \mathbf{n}}=t \text { on } \Gamma_{N},
\end{aligned}
$$

where $u$ is the scalar quantty to be transported and also the unknown field of the problem, $\mathbf{a}$ is the convection velocity, $\nu>0$ the diffusivity, assumed constant and $s(\mathbf{x})$ a volumetric source term.

The function $u_{D}$ denotes the prescribed value of $u$ on the Dirichlet boundary, given by $\Gamma_{D}$, the function $u_{N}$ denotes the value of the normal diffusive flux on the Neumann boundary $\Gamma_{N}$.

The weak form of the problem given by the previous equations is Find $u(\mathbf{x}) \in \mathcal{S}=\left\{u \in \mathcal{H}^{1}(\Omega) \mid u=u_{D}\right.$ on $\left.\Gamma_{D}\right\}$ such that $\forall w \in \mathcal{V}=\left\{w \in \mathcal{H}^{1}(\Omega) \mid w=0\right.$ on $\left.\Gamma_{D}\right\}$

$$
\int_{\Omega} w(\mathbf{a} \cdot \nabla u) d \Omega+\int_{\Omega} \nabla w \cdot(\nu \nabla u) d \Omega=
$$




$$
=\int_{\Omega} w s d \Omega+\int_{\Gamma_{N}} w t d \Gamma
$$

that expressed in a compact form, we obtain,

$$
a(w, u)+c(\mathbf{a} ; w, u)=(w, s)+(w, t)_{\Gamma_{N}}
$$

\subsection{CONSISTENT, SUPG, STABILIZATION}

The general form of the consistent stabilization techniques is [1]

$$
\begin{array}{r}
a(w, u)+c(\mathbf{a} ; w, u)+\underbrace{\sum_{\Gamma_{N}} \int_{\Omega_{e}} \mathcal{P}(w) \tau \mathcal{R}(u) d \Omega}_{\begin{array}{c}
\text { stabilization term } \\
=(w, s)+(w, t)
\end{array}}= \\
=
\end{array}
$$

where $\mathcal{P}(w)$ is an operator applied to the test function and taking by $\mathcal{P}(w)=\mathbf{a} \cdot \nabla w$ in the SUPG stabilization technique, which, in fact, corresponds to the perturbation of the test function. The stabilization parameter $\tau=\frac{h}{2 a}\left(\operatorname{coth} P e-\frac{1}{P e}\right)$ is called intrinsic time and this expression provides us the exact nodal values for 1D convection-diffusion equation, where $P e$ is the Pclet number defined as $P e=a h / 2 \nu$. $h$ represents the mesh size parameter and $a$ the modulus of the convective velocity. Finally, $\mathcal{R}(u)$ is the residual of the differential equation. In this paper we introduce a stabilization technique based upon the use of the so-called Proper Generalized Decompositions (PGD) introduced in [2] and [3].

\section{THE METHOD}

In this section we explain briefly the method based on the method of separation of variables, originally developed in [2].

For simplicity, we start by considering the Poisson problem defined in a space of dimension $\mathrm{N}$ with homogeneous boundary conditions,

$$
-\Delta u=f\left(x_{1}, x_{2}, \ldots, x_{N}\right) \text { in } \Omega=(-L, L)^{N},
$$

The problem solution can be written in the form

$$
T\left(x_{1}, x_{2}, \ldots, x_{N}\right)=\sum_{j=1}^{Q} \alpha_{j} \prod_{k=1}^{N} F_{k j}\left(x_{k}\right),
$$

where $F_{k j}$ is the $j$ th basis function, with unit norm, and only depends on the $k$ th coordinate. Note that the solution of numerous problems can be accurately approximated using a finite number $Q$ of approximation functions.

The numerical scheme proposed consist of an iteration procedure that solves at each iteration $n$ the following three steps.

Step 1 A projection step of the solution in a discrete basis, where the coefficients $\alpha_{j}$ are computed.

Step 2 Checking convergence step.
Step 3 Enrichment of the approximation basis step. From the coefficients $\alpha_{j}$ just computed the approximation basis can be enriched by adding the new function $\prod_{k=1}^{N} F_{k(n+1)}\left(x_{k}\right)$.

For details of this scheme see [2].

\subsection{APPLICATION TO THE CONVECTION- DIFFUSION EQUATION}

In the enrichment stage we look for an improved representation of the essential field in the form

$$
\begin{array}{r}
u^{h}(x, y)=\sum_{i=1}^{n} \alpha_{i} F_{i}(x) \cdot G_{i}(y)+R(x) \cdot S(y)= \\
=u^{H}(x, y)+R(x) \cdot S(y)
\end{array}
$$

The test function will then be given by

$$
w^{*}(x, y)=R^{*}(x) \cdot S(y)+R(x) \cdot S^{*}(y) .
$$

Applying these definitions to the weak form (6), we obtain

$$
\begin{array}{r}
a\left(R^{*} S+R S^{*}, R S\right)+c\left(\mathbf{a} ; R^{*} S+R S^{*}, R S\right)= \\
=-a\left(R^{*} S+R S^{*}, u^{H}\right)-c\left(\mathbf{a} ; R^{*} S+R S^{*}, u^{H}\right)+ \\
+l\left(R^{*} S+R S^{*}\right)- \\
-\sum_{e} \int_{\Omega_{e}} \mathbf{a} \nabla\left(R^{*} S+R S^{*}\right) \tau\left[\mathcal{L}\left(u^{H}\right)-s\right] d \Omega
\end{array}
$$

After applying such an approximation, the weak form of the problem given by (3.1) is solved by some kind of iterative procedure, such as Newton-Raphson, alternating directions fixed-point algorithm, etc.

\section{NUMERICAL EXAMPLE}

The problem statement is depicted in Figure 1, where the unit square is taken as the computational domain, $\bar{\Omega}=[0,1] \times[0,1]$. This $2 \mathrm{D}$ test case has been widely

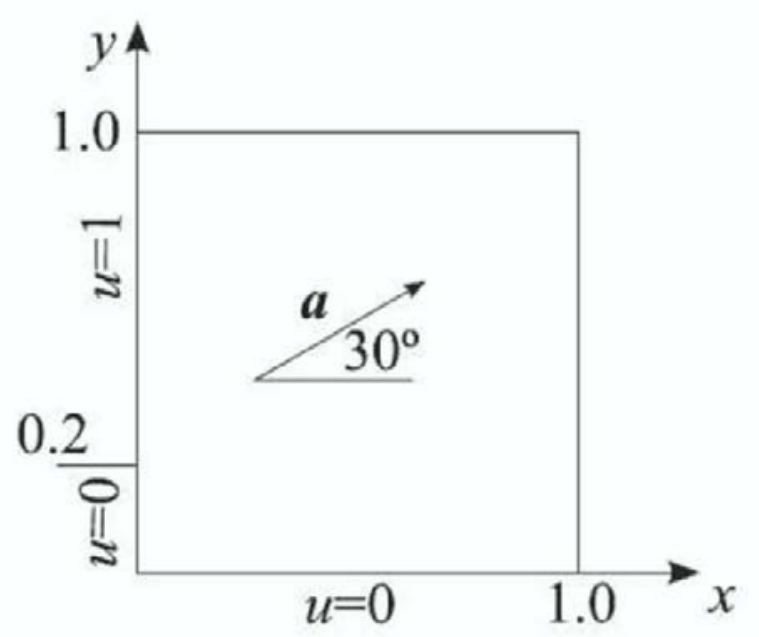

Figure 1: Convection of discontinuous inlet data skew to the mesh: problem statement. 
used to illustrate the effectiveness of stabilized finite element methods in the modelling of convection-dominated flows. A mesh of 10 by 10 equal-size bilinear elements is considered in the standard finite element approach, only a 10 elements along each direction is used in the PGD framework.

The flow is unidirectional and constant, $\|\mathbf{a}\|=1$, but the convective velocity is skew to the mesh with an angle of 30 . The diffusivity coefficient is taken to be $5 \cdot 10^{-6}$, corresponding to a Pclet number of $10^{4}$. The inlet boundary data are discontinuous and two types of boundary are considered at the outlet:

- Downwind homogeneous natural boundary conditions. The results for this case are displayed in Figure 2. Given the elevated value of the Pclet number, the solution is practically one of pure convection.
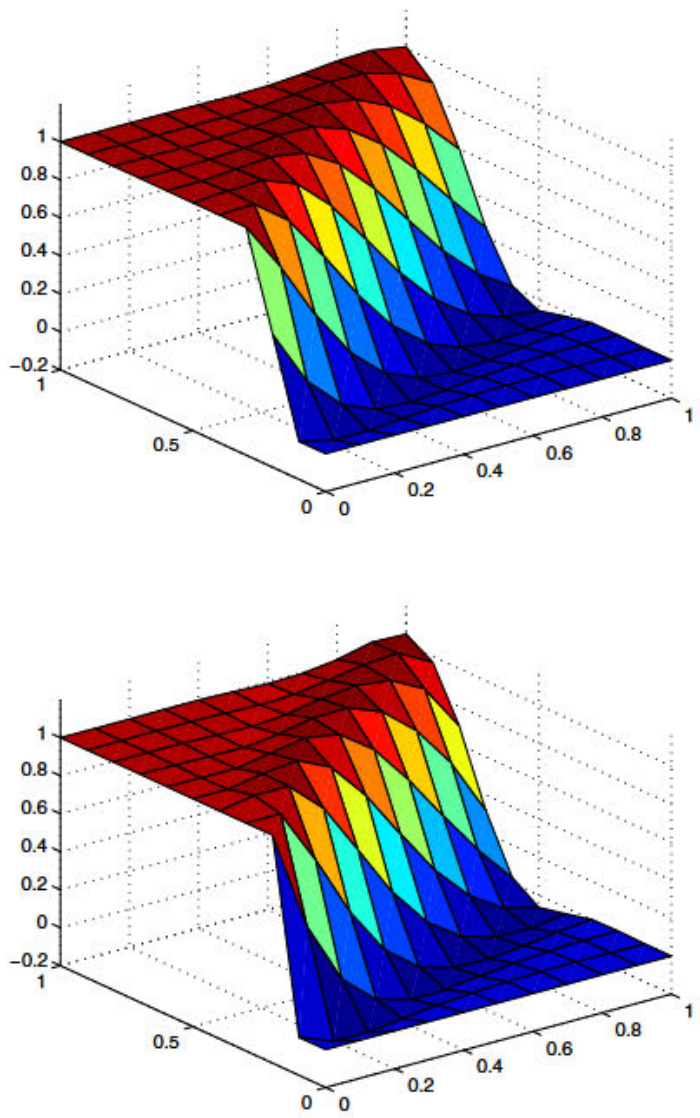

Figure 2: Results for the Neumann Boundary Conditions: MEF-SUPG(up) PGD-SUPG(down).

In the Figure 3 we can see a cross along $x=0.5$ of both approach.

- Downwind homogeneous essential boundary conditions. Here we impose $u=0$ on the outlet portion of the boundary. The results are depicted in the Figure 4.

In the Figure 5 we can see a cross along $x=0.5$ of both approach.

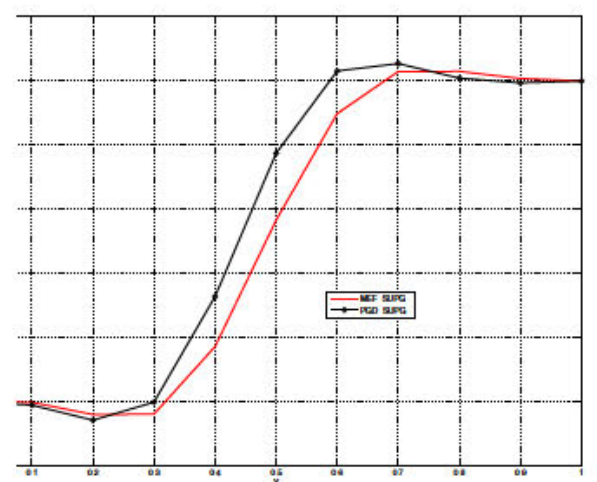

Figure 3: Cross along $x=0.5$ for the Neumann boundary conditions problem.

\section{CONCLUSIONS}

We have presented a technique of separated representations (also coined recently Proper Generalized Decompositions) for the stabilization of convection-diffusion equations solved by finite elements. The key characteristic of this technique is the representation of the essential field in terms of a finite sum of separable functions, leading to a set of one-dimensional SUPG problems, therefore with exact stabilization known.

The separated representations also allow for an important saving in terms of degrees of freedom, which is particularly important for problems defined in spaces of high number of dimensions. It has been show how this approximation leads to more stable results than the traditional SUPG technique.

\section{REFERENCES}

[1] J. Donea and A. Huerta. Finite Element Methods for Flow Problems. John Wiley and sons, 2003.

[2] F. Chinesta A. Ammar, B. Mokdad and R. Keunings. A New Family of Solvers for Some Classes of Multidimensional Partial Differential Equations Encountered in Kinetic Theory Modeling of Complex Fluids. Journal of Non-Newtonian Fluid Mechanics, 139 (3):153-176, 2006.

[3] F. Chinesta A. Ammar, B. Mokdad and R. Keunings. A New Family of Solvers for Some Classes of Multidimensional Partial Differential Equations Encountered in Kinetic Theory Modeling of Complex Fluids. Part II: transient simulation using space-time separated representations. Journal of Non-Newtonian Fluid Mechanics, 144:98-121, 2007. 
